\title{
Not Just for Trauma Patients: Damage Control Laparotomy in Pancreatic Surgery (Response to Letter to the Editor)
}

\author{
Katherine Morgan • David B. Adams
}

Received: 26 May 2010 / Accepted: 30 June 2010/Published online: 30 July 2010

(C) 2010 The Society for Surgery of the Alimentary Tract

We appreciate your excellent commentary on damage control surgery and particularly the appropriate acknowledgement of the icons of modern trauma surgery.

The magnitude of your experience with acute pancreatitis at Indiana is as always impressive. This large volume makes your descriptive data an important contribution to the accurate understanding of this challenging disease. Certainly, much can be learned from your conduct in the management of these ill patients. It is notable that we excluded patients undergoing laparostomy or debridement for severe acute pancreatitis with necrosis from our report on elective pancreas surgery. The inflammatory process is a likely contributor to the physiologic derangements surrounding perioperative events. Presumably, patients with necrotizing pancreatitis are a sicker subgroup of pancreas surgery patients.

The trauma literature is robust with helpful guidelines for the institution of damage control surgery, given the large numbers of evaluable patients. The criteria for application of damage control in the previously healthy trauma warrior, however, are potentially quite different from the criteria for its optimal use in a patient with catastrophe while undergoing elective pancreatic surgery, with the attendant metabolic disadvantage of chronic illness (cancer or pancreatitis) and the resultant limited physiologic reserve. Therefore, the appropriate parameters for applying damage control in the pancreas surgery patient are currently uncertain, and the judgment of the experienced pancreatic surgeon remains important.

We reported $\mathrm{pH}$ as a marker of acidosis. The median base deficit in our series was 11.1 (range 0.4 to 16.6). The interval to abdominal closure in those patients who achieved it was a median of 3 days (range 1 to 3 days). Patient \#7 was relegated to damage control due to intraoperative coagulopathy from sepsis. Patient \#8 underwent damage control as a result of intraoperative acidosis and coagulopathy also due to sepsis.

We greatly appreciate your thoughtful comments and common interest in taking optimal care of the pancreas surgery patient. Given the relatively rare need for damage control in elective pancreas surgery, perhaps more experience or more likely a collaborative effort could help to more precisely define the role of damage control.
K. Morgan $(\bowtie) \cdot$ D. B. Adams

Medical University of South Carolina,

Charleston, SC, USA

e-mail: morganka@musc.edu 